\title{
Quantitative lipid profiling of the early response to either fructose or glucose in an in vitro model of steatosis
}

\author{
E.M. Maldonado, B.A. Fielding, C.P. Fisher and J.B. Moore \\ School of Biosciences and Medicine, University of Surrey, Guildford, Surrey, GU2 7XH
}

Whether or not dietary sugars, including fructose, exacerbate hepatic lipogenesis and non-alcoholic fatty liver disease (NAFLD) pathogenesis remains an unresolved question. Here, we quantitatively assess the early metabolic response to dietary sugars in oleic acid (OA) treated HepG2 cells, a 'mixed nutrient' model of NAFLD.

HepG2 cells were sub-cultured in medium containing physiological levels of glucose $(5 \mathrm{mM}, 5 \mathrm{G})$, then treated with vehicle $(\mathrm{V}+5 \mathrm{G})$, $400 \mu \mathrm{M}$ oleic acid $(\mathrm{OA}+5 \mathrm{G})$ or OA with an additional $20 \mathrm{mM}$ of monosaccharide as either glucose $(\mathrm{OA}+25 \mathrm{G})$ or fructose $(\mathrm{OA}+5 \mathrm{G}+$ $20 \mathrm{~F}$ ). Intracellular lipid was quantified at $0,2,4,6$ and $24 \mathrm{~h}$ time points. Nile red, a neutral lipophilic fluorescent dye, was used to measure total intracellular lipid and quantified either relative to vehicle or from an olive oil standard curve. Gas chromatography mass spectrometry (GC/MS) was used to measure intracellular lipid after Folch extraction with spiked internal standards. The extracted lipid was separated into fractions (i.e. diacylglycerol, DAG and triacylglycerol, TAG) by thin-layer chromatography before quantification of fatty acids (e.g. palmitic acid, 16:0 and oleic acid, 18:1n9) in each fraction. Data in figure 1 were statistically analysed by two-way ANOVA with Tukey's test post hoc.

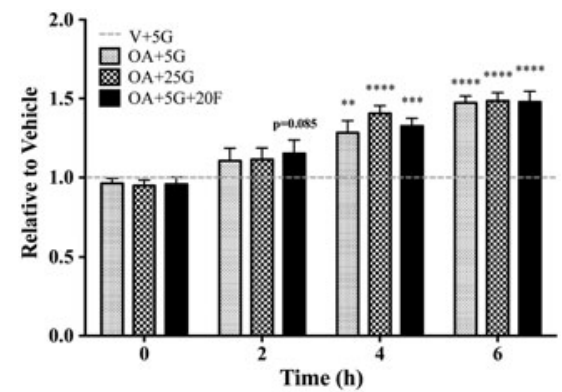

Fig. 1. Relative intracellular lipid at $24 \mathrm{~h}$.

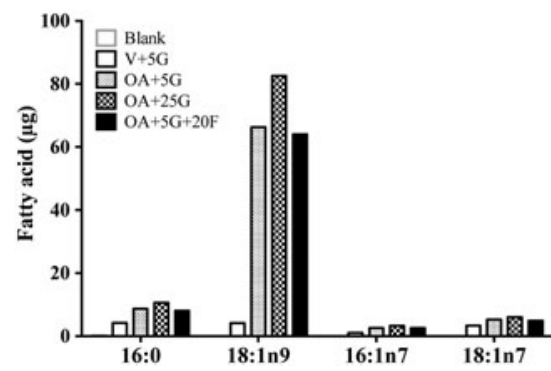

Fig. 2. TAG fraction at $6 \mathrm{~h}$.

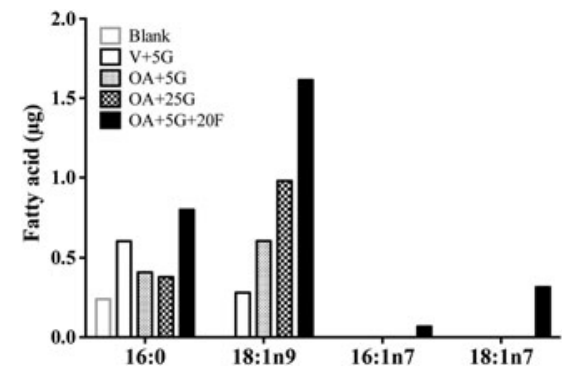

Fig. 3. DAG fraction at $6 \mathrm{~h}$.

After a $24 \mathrm{~h}$ treatment with OA in $5 \mathrm{mM}$ and $25 \mathrm{mM}$ glucose, there was no difference in total intracellular lipid as detected by nile red. This held true when an olive oil standard curve was used $(n=4$, data not shown). However, at earlier time points, the rate of lipid accumulation differed between treatments as detected by nile red $(n=4-5$, statistical differences indicated as time point versus 0 , Fig. 1). When assessed by quantitative GC/MS, initial results show a higher accumulation of TAG in OA $+25 \mathrm{G}$ in comparison to $\mathrm{OA}+5 \mathrm{G}$ and $\mathrm{OA}+5 \mathrm{G}+20 \mathrm{~F}$ after $6 \mathrm{~h}$ of treatment; in particular, oleic acid in the TAG fraction $(\mathrm{n}=1$, Fig. 2$)$. Interestingly, a higher amount of DAG was detected in $\mathrm{OA}+5 \mathrm{G}+20 \mathrm{~F}$ treated cells in comparison to OA + 25G; furthermore, higher amounts of palmitic acid, oleic acid and the de novo lipogenesis associated fatty acids, 16:1n7 and 18:1n7, were also detected (n=1, Fig. 3).

Data regarding a differential role for dietary sugars in liver fat accumulation and NAFLD pathogenesis is somewhat conflicted. A recent meta-analysis of human intervention trials shows no difference in the extent of fat accumulation in the liver from equimolar doses of fructose or glucose ${ }^{(1)}$. However, our data shows an initial increase in DAG synthesis in response to co-treatment with lipid and fructose; highlighting downstream signalling pathways fructose may modulate. Future studies will explore responses in the presence of insulin and monitor early signalling responses to fatty acids and sugars using proteomic approaches.

1. Chiu S, Sievenpiper JL, de Souza RJ et al. (2014) Eur J Clin Nutr 86, 416-23. 